\title{
Valorization of Vegetable Food Waste and By-Products Through Fermentation Processes
}

\author{
Carlos Sabater,2*, Lorena Ruiz ${ }^{1,2}$, Susana Delgado 1,2, Patricia Ruas-Madiedo ${ }^{1,2}$ and \\ Abelardo Margolles ${ }^{1,2}$ \\ ' Department of Microbiology and Biochemistry of Dairy Products, Instituto de Productos Lácteos de Asturias, Consejo \\ Superior de Investigaciones Científicas, Villaviciosa, Spain, ${ }^{2}$ Instituto de Investigación Sanitaria del Principado de Asturias, \\ Oviedo, Spain
}

\section{OPEN ACCESS}

Edited by:

Stavros Plessas,

Democritus University of Thrace,

Greece

Reviewed by:

Erica Pontonio,

University of Bari Aldo Moro, Italy

Yiannis Kourkoutas,

Democritus University of Thrace,

Greece

*Correspondence:

Carlos Sabater

carlos.sabater@csic.es

Specialty section:

This article was submitted to

Food Microbiology,

a section of the journal

Frontiers in Microbiology

Received: 10 July 2020

Accepted: 28 September 2020

Published: 20 October 2020

Citation:

Sabater C, Ruiz L, Delgado S, Ruas-Madiedo $P$ and Margolles A (2020) Valorization of Vegetable Food Waste and By-Products Through

Fermentation Processes.

Front. Microbiol. 11:581997. doi: 10.3389/fmicb.2020.581997
There is a general interest in finding new ways of valorizing fruit and vegetable processing by-products. With this aim, applications of industrial fermentation to improve nutritional value, or to produce biologically active compounds, have been developed. In this sense, the fermentation of a wide variety of by-products including rice, barley, soya, citrus, and milling by-products has been reported. This minireview gives an overview of recent fermentation-based valorization strategies developed in the last 2 years. To aid the designing of new bioprocesses of industrial interest, this minireview also provides a detailed comparison of the fermentation conditions needed to produce specific bioactive compounds through a simple artificial neural network model. Different applications reported have been focused on increasing the nutritional value of vegetable by-products, while several lactic acid bacteria and Penicillium species have been used to produce high purity lactic acid. Bacteria and fungi like Bacillus subtilis, Rhizopus oligosporus, or Fusarium flocciferum may be used to efficiently produce protein extracts with high biological value and a wide variety of functional carbohydrates and glycosidases have been produced employing Aspergillus, Yarrowia, and Trichoderma species. Fermentative patterns summarized may guide the production of functional ingredients for novel food formulation and the development of low-cost bioprocesses leading to a transition toward a bioeconomy model.

Keywords: food waste, microbial fermentation, by-product Valorization, lactic acid bacteria, bioeconomy

\section{INTRODUCTION}

The plant-based food manufacturing sector is currently under expansion and is generating large amounts of by-products. Improved waste management to mitigate the negative environmental impacts of fruit and vegetable processing industries is an essential step in the transition toward a bioeconomy. Therefore, there is a common interest in finding new ways to valorize these substrates, and green valorization schemes that lead to an integrated biorefinery platform have been introduced (Satari and Karimi, 2018). Industrial by-products derived from agriculture, forestry and fishing comprise 1.4\% of the total waste production in the European Union (Bedoić et al., 2019). Agricultural waste, co-products and by-products play a relevant role in animal feed production worldwide, while biomass residues are widely used in the production of bioenergy. However, 
a sustainable bioeconomy must prioritize the production of highquality foods (Valenti et al., 2020). The generation of these residues strongly depends on land activities, climate conditions, and consumption of goods. It has been reported that Western European countries show a high potential for the use of byproducts from vegetable cultivation activities. On the other hand, high volumes of cereal by-products are obtained in Central and Eastern Europe. Valorization of citrus by-products could be of great interest in Southern European countries due to their land areas and mild weather conditions (Bedoić et al., 2019; Valenti et al., 2020), and bioeconomy models for bioenergy generation from Mediterranean feedstocks have been developed (Valenti et al., 2020).

More innovative valorization strategies aim to recover high value ingredients from fruit and vegetable by-products, used as natural sources of biologically active compounds for drug and functional food formulation (Lu et al., 2019). With this aim, fermentative processes using lactic acid bacteria (LAB) and other microorganisms have been described. Fermentative processes can be classified according to different criteria. Most applications developed involve batch fermentations where the substrate and producing microorganism are added to the system at time zero and are not removed until the process is complete. In contrast, in continuous and fed-batch fermentations microorganisms may be immobilized and reutilized for several cycles leading to higher efficiency (Burke et al., 2013). While many industrial fermentations take place in liquid media, in solid-state fermentations microorganisms are grown on a solid support. Shake flasks are the most popular reaction vessels for bioprocessing due to their simplicity, and one-step fermentations have been reported as enhancing mass transfer and reducing potential inhibition by the substrate (Amorim et al., 2019).

Recent examples of fermentation-based valorization strategies include anaerobic digestion of organic feedstocks through mixed culture fermentation (De Groof et al., 2019), fermentation of date palm waste to produce lactic acid as an alternative for the expensive raw material (Azam and Ahmad, 2019), bioconversion of cocoa by-products using bacteria, yeast or filamentous fungi to obtain enzymes, polysaccharides, beverages and nutraceuticals (Vásquez et al., 2019), and sourdough fermentation to elaborate baked foods that may ameliorate the symptoms of irritable bowel syndrome and release bioactive compounds related to the metabolism of phenolic compounds (Gobbetti et al., 2019). In general, most of the applications mentioned above use $\mathrm{LAB}$ to improve the nutritional value of several food matrices, or to isolate biologically active ingredients for functional food formulation. Nevertheless, bioconversion processes and fermentation strategies to increase digestibility, enhance nutritional value and decrease the levels of antinutritional factors in these substrates using other types of bacteria, as well as different yeasts and molds, have been described (Chebaibi et al., 2019; De Groof et al., 2019; Lücke et al., 2019; Vásquez et al., 2019).

The first part of this minireview provides an overview of recent trends in by-product valorization through microbial fermentation considering: (i) microorganisms selected (LAB, other bacteria, fungi, and yeasts), (ii) main compounds produced and nutritional achievements, (iii) fruit and vegetable wastes and by-products. Then, a comparison of fermentation conditions that should be selected depending on the application as well as a tentative consideration of their economic feasibility are presented.

To select the articles considered in this minireview, we mainly focused on the papers published in the last 2 years and listed in the Web Of Science that were searched using the following keywords and terms: "fermentation," "vegetable," and "by-product" or "waste." Results were then filtered and only research articles reporting fermentation conditions were chosen.

\section{FERMENTATION PROCESSES EMPLOYING LAB}

Lactic acid bacteria (LAB) constitute a group of microorganisms of great industrial interest since they are involved in the production of many fermented foods from raw materials of animal (mainly milk) and vegetable origin (Torres et al., 2020), as well as in feed silage fermentations (Avila and Carvalho, 2020). LAB are highly specialized in the bioconversion of the carbohydrates in lactic acid, rending as well minor amounts of other organic acids which reduce the $\mathrm{pH}$, and are a natural way of conservation; the metabolic activity of LAB on other substrates also have a deep impact in the sensorial, technological, nutritional, and functional characteristics of the resulting fermented foods and feeds (Brückner-Gühmann et al., 2019; Kimoto-Nira et al., 2019; Schettino et al., 2019). The traditional ways of preserving raw materials have favored the "domestication" or selection of specific bacterial lineages well-adapted to the fermented products (Gibbons and Rinker, 2015; Li and Gänzle, 2020). Currently, from this empirical, or non-intentioned, use of LAB comes the application of starter and adjunct cultures from controlled fermentations in the manufacture of a wide variety of fermented foods.

The LAB cultures commonly used in controlled food manufacture are Streptococcus thermophilus, Lactococcus lactis, Leuconostoc spp. and Lactobacillus spp., for dairy products, but also the genera Pediococcus, Oenococcus, and Weissella play a pivotal role in plant-based fermented products (Wuyts et al., 2020). Leading approaches are used to improve the characteristics of these industrial bacteria (Hidalgo-Cantabrana et al., 2017; Bron et al., 2019), but the natural resources are also relevant to find novel strains with biotechnological applications (Bachtarzi et al., 2019; Petrova and Petro, 2020). Therefore, the same approaches can be used for the selection of the best LAB candidates to ferment residues from plant materials. Valorization strategies using $\mathrm{LAB}$ include the production of lactic acid that may be reintegrated in the food chain as well as enhancing protein digestibility and sensorial properties of these vegetable by-products that could be used as food ingredients (Table 1).

As indicated above, the agro-industrial activity generates a wide diversity of waste which is also susceptible to be spontaneously fermented by the microbiota naturally present in these by-products (Verni et al., 2019). Additionally, directed and controlled fermentations can also be driven in order to 
TABLE 1 | By-product and waste valorization through fermentative processes and enzymatic hydrolysis.

\begin{tabular}{|c|c|c|c|c|c|}
\hline Substrate & Classification & $\begin{array}{l}\text { Type of } \\
\text { microorganism }\end{array}$ & Main results & $\begin{array}{l}\text { Reintegration in } \\
\text { the food chain }\end{array}$ & References \\
\hline Rice pasta & By-product & Fungi & Compound production & Yes & Jirasatid et al., 2019 \\
\hline Defatted rice bran & By-product & Bacteria & Compound production & Yes & Alexandri et al., 2019 \\
\hline Rice husk & By-product & $\begin{array}{l}\text { Fungi, yeast, } \\
\text { and bacteria }\end{array}$ & Compound production & Yes & Montipó et al., 2019 \\
\hline Rice kernel & By-product & Bacteria & Compound production & Yes & Saman et al., 2019 \\
\hline Rice straw, husk, and bran & By-product & Fungi & Compound production & Yes & Postemsky et al., 2019 \\
\hline Brewer's spent grain & By-product & Fungi & Compound production & Yes & Amorim et al., 2019 \\
\hline Brewer's spent grain & By-product & Fungi & Compound production & No & Outeiriño et al., 2019 \\
\hline Brewer's spent grain & By-product & Fungi & Compound production & Yes & Paz et al., 2019 \\
\hline Brewer's spent yeast & By-product & Bacteria & Compound production & Yes & Pejin et al., 2019 \\
\hline Brewer's spent yeast & By-product & $\begin{array}{l}\text { Fungi and } \\
\text { bacteria }\end{array}$ & Enhanced properties & Yes & Marson et al., 2019 \\
\hline Barley brans & By-product & Bacteria & Enhanced properties & Yes & Pontonio et al., 2020 \\
\hline Soybean dregs & By-product & Bacteria & Compound production & Yes & Jiang et al., 2019 \\
\hline Soybean meal & By-product & Bacteria & Compound production & Yes & Mukherjee et al., 2019 \\
\hline Soybean meal & By-product & Bacteria & Compound production & Yes & Ruan et al., 2020 \\
\hline Okara (from soymilk) & By-product & Bacteria & Compound production & No & Orts et al., 2019 \\
\hline Soy whey & By-product & Yeast & Compound production & Yes & Chua and Liu, 2020 \\
\hline Soybean cake & By-product & Yeast & Compound production & Yes & Papadaki et al., 2019 \\
\hline Soybean hulls/wheat bran & By-product & Fungi & Compound production & No & Taddia et al., 2019 \\
\hline Wheat bran & By-product & Bacteria & Enhanced properties & Yes & Spaggiari et al., 2020 \\
\hline Pineapple peels & Waste & Fungi & Enhanced properties & Yes & Aruna, 2019 \\
\hline Apple by-products & By-product & $\begin{array}{l}\text { Bacteria and } \\
\text { yeast }\end{array}$ & Enhanced properties & Yes & Cantatore et al., 2019 \\
\hline Orange peels & By-product & Bacteria & Compound production & Yes & Ricci et al., 2019b \\
\hline Mandarin orange waste & Waste & Bacteria & Enhanced properties & No & Tomita et al., 2019 \\
\hline Citrus depressa pomace & By-product & Bacteria & Enhanced properties & Yes & Kimoto-Nira et al., 2019 \\
\hline Mango seed & By-product & Fungi & Enhanced properties & Yes & Torres-León et al., 2019 \\
\hline Blueberry pomace & By-product & Bacteria & Enhanced properties & Yes & Cheng et al., 2020 \\
\hline Grape pomace flour & By-product & Fungi & Compound production & Yes & Costa et al., 2019 \\
\hline Melon/Tomato/Carrot & By-product & Bacteria & Enhanced properties & Yes & Ricci et al., 2019a \\
\hline Fruit and vegetable wastes & Waste & Bacteria & Compound production & No & Yu et al., 2019 \\
\hline Sweet potato distillery by-product & By-product & Fungi & Enhanced properties & Yes & Kosakai et al., 2019 \\
\hline Molasses/potato stillage & By-product & Bacteria & Compound production & Yes & Mladenović et al., 2019a \\
\hline Molasses/potato stillage & By-product & Bacteria & Compound production & Yes & Mladenović et al., 2019b \\
\hline Cane molasses & By-product & Yeast & Compound production & Yes & Wang et al., 2019 \\
\hline Maize milling by-products & By-product & Bacteria & Enhanced properties & Yes & Pontonio et al., 2019 \\
\hline Lime cooked maize by-product & By-product & Fungi & Enhanced properties & Yes & Acosta-Estrada et al., 2019 \\
\hline Olive cake & By-product & Fungi & Enhanced properties & Yes & Chebaibi et al., 2019 \\
\hline Olive-mill wastewaters & Waste & Yeast & Compound production & Yes & Sarris et al., 2019 \\
\hline Rapeseed presscake & By-product & Fungi & Enhanced properties & Yes & Lücke et al., 2019 \\
\hline Argan press cake-suspension & Waste & Bacteria & Compound production & Yes & Goto et al., 2019 \\
\hline Hemp/chickpea milling by-products & By-product & Bacteria & Enhanced properties & Yes & Schettino et al., 2019 \\
\hline Grain sorghum flour & By-product & $\begin{array}{l}\text { Fungi and } \\
\text { bacteria }\end{array}$ & Enhanced properties & No & Cole et al., 2019 \\
\hline Cassava flour by-product & By-product & Bacteria & Compound production & Yes & do Carmo Brito et al., 2019 \\
\hline Cold-pressing oil products & By-product & Fungi & Compound production & No & Tišma et al., 2019 \\
\hline Oat protein concentrate & By-product & Bacteria & Enhanced properties & Yes & Brückner-Gühmann et al., 2019 \\
\hline Carob pod waste & Waste & Bacteria & Compound production & Yes & Bahry et al., 2019 \\
\hline Aguamiel from agave & By-product & Fungi & Compound production & Yes & Muñiz-Márquez et al., 2019 \\
\hline
\end{tabular}


TABLE 1 | Continued

\section{Species and strains}

Monascus purpureus TISTR 3541/Monascus purpureus TISTR 3629

Bacillus coagulans A107

Penicillium echinulatum S1M29/Lactobacillus buchneri NRRL

B-30929/Saccharomyces cerevisiae CAT-1

Lactiplantibacillus plantarum NCIMB 8826/Limosilactobacillus reuteri NCIMB

8821

Pleurotus sapidus MkP6

Trichoderma reesei

Aspergillus brasiliensis CECT 2700

Aspergillus niger CECT 2700

Lacticaseibacillus rhamnosus ATCC 7469

Aspergillus oryzae/Bacillus subtilis/Bacillus licheniformis

Lactiplantibacillus plantarum T6B10/Weissella confusa BAN8

Bacillus amyloliquefaciens NX-2S

Bacillus coagulans NCIM 2323/Lactobacillus johnsonii LMG 18175

Bacillus subtilis

Bacillus licheniformis ATCC 21415

Torulaspora delbrueckii

Rhodosporidium toruloides

Aspergillus niger NRRL3

Lacticaseibacillus rhamnosus 1473

Trichoderma viride ATCC 36316

Weissella cibaria PEP23F/Saccharomyces cerevisiae AN6Y19

Lacticaseibacillus casei 2246

Clostridium beijerinckii NCIMB8052/Clostridium cellulovorans 743B

Lactiplantibacillus plantarum E58

Aspergillus niger $\mathrm{GH} 1$

Lacticaseibacillus casei

Aspergillus niger 3T5B8/Aspergillus aculeatus

Lactiplantibacillus plantarum/Lacticaseibacillus casei/Lacticaseibacillus

paracasei/Lacticaseibacillus rhamnosus

Predominantly Clostridium kluyveri

Aspergillus oryzae

Lacticaseibacillus paracasei NRRL B-4564

Lacticaseibacillus paracasei NRRL B-4564

Yarrowia lipolytica S47

Lactiplantibacillus plantarum T6B10/Weissella confusa BAN8

Aspergillus oryzae/Pleurotus ostreatus/Hericium erinaceus

Aspergillus niger/Beauveria bassiana/Fusarium flocciferum/Rhizodiscina cf. lignyota

Yarrowia lipolytica ACA-DC 5029

Rhizopus microsporus var. oligosporus

Lactiplantibacillus plantarum Argan-L1

Lactiplantibacillus plantarum LB1/Furfurilactobacillus rossiae LB5

Bacillus licheniformis/Bacillus subtilis/Aspergillus niger/Aspergillus aculeatus

Lactiplantibacillus plantarum/Lactobacillus delbrueckii/Limosilactobacillus

fermentum

Thermomyces lanuginosus

Lactobacillus delbrueckii subsp. bulgaricus/Streptococcus thermophilus

Lacticaseibacillus rhamnosus

Aspergillus niger GH1/Aspergillus niger PSH/Aspergillus oryzae DIA-MF

Compounds produced /
nutritional achievements

achievements

Pigments

Lactic acid

Lactic acid

Lactic acid

Nanocellulose

Arabinoxylo-oligosaccharides

Glycosidases

Xylose

Lactic acid

Protein content

Protein digestibility

Poly- $\gamma$-glutamic acid

Antioxidant activity

Antihypertensive peptides

Glycosidases

Organic acids

Carotenoids

Glycosidases

Arabinoxylan solubility

Protein content

Fiber content

Lactic acid

Sugar content

Enhanced sensorial properties

Antioxidant activity

Phenolic compounds

Xylo-oligosaccharides

Antimicrobial activity

Caproate

Microbiota modulation:

production of SCFAs

Lactic acid

Lactic acid

Isomaltulose

Protein digestibility

Fiber content

Protein content

Citric and oleic acid

Fiber and protein content

Lactic acid

Fiber and protein content

Increase fermentable sugars

Lactic acid

Lipase

Technological properties

Lactic acid

Fructo-oligosaccharides
Yield / increment

(+) / decrease (-)

880 OD units/g

$900 \mathrm{mg} / \mathrm{g}$

$533 \mathrm{mg} / \mathrm{g}$

$0.7 \mathrm{~g} / \mathrm{L}$

$670 \mathrm{mg} / \mathrm{g}$

$38 \mathrm{mg} / \mathrm{g}$

$3152 \mathrm{U} / \mathrm{g}$

$6 \mathrm{~g} / \mathrm{L}$

$890 \mathrm{mg} / \mathrm{g}$

$+50 \%$

$+87 \%$

$66 \mathrm{mg} / \mathrm{g}$

$73 \%$

$89 \mathrm{mg} / \mathrm{g}$

$2 \mathrm{U} / \mathrm{g}$

$6 \mathrm{~g} / \mathrm{L}$

$89 \mu \mathrm{g} / \mathrm{g}$

$555 \mathrm{U} / \mathrm{mL}$

$+200 \%$

$+15 \%$

$+40 \%$

$880 \mathrm{mg} / \mathrm{g}$

$-85 \%$

$+56 \%$

$+90 \%$

$0.5 \mathrm{mg} / \mathrm{mL}$

$887 \mathrm{mg} / \mathrm{g}$

$+700 \%$

$620 \mathrm{mg} / \mathrm{g}$

$+239 \%$

$399 \mathrm{~g} / \mathrm{L}$

$890 \mathrm{mg} / \mathrm{g}$

$960 \mathrm{mg} / \mathrm{g}$

$+60 \%$

$+45 \%$

$+94 \%$

$550 \mathrm{mg} / \mathrm{g}$

$+11 \%$

$5 \mathrm{~g} / \mathrm{L}$

$+13 \%$

$200 \%$

$13 \mathrm{~g} / \mathrm{L}$

$60 \mathrm{U} / \mathrm{mL}$

$+96 \%$

$22 \mathrm{~g} / \mathrm{L}$

$470 \mathrm{mg} / \mathrm{g}$
References

Jirasatid et al., 2019

Alexandri et al., 2019

Montipó et al., 2019

Saman et al., 2019

Postemsky et al., 2019

Amorim et al., 2019

Outeiriño et al., 2019

Paz et al., 2019

Pejin et al., 2019

Marson et al., 2019

Pontonio et al., 2020

Jiang et al., 2019

Mukherjee et al., 2019

Ruan et al., 2020

Orts et al., 2019

Chua and Liu, 2020

Papadaki et al., 2019

Taddia et al., 2019

Spaggiari et al., 2020

Aruna, 2019

Cantatore et al., 2019

Ricci et al., 2019a

Tomita et al., 2019

Kimoto-Nira et al., 2019

Torres-León et al., 2019

Cheng et al., 2020

Costa et al., 2019

Ricci et al., 2019b

Yu et al., 2019

Kosakai et al., 2019

Mladenović et al., 2019a

Mladenović et al., 2019b

Wang et al., 2019

Pontonio et al., 2019

Acosta-Estrada et al., 2019

Chebaibi et al., 2019

Sarris et al., 2019

Lücke et al., 2019

Goto et al., 2019

Schettino et al., 2019

Cole et al., 2019

do Carmo Brito et al., 2019

Tišma et al., 2019

Brückner-Gühmann et al., 2019

Bahry et al., 2019

Muñiz-Márquez et al., 2019

SCFAs, short-chain fatty acids; $U$, enzyme activity units; OD, optical density units. 
obtain valorized final products; in spite of the low predominance of LAB in plant autochthonous microbiota, they can also be key players in plant and plant-derived waste fermentations (Filannino et al., 2018). The reason behind this is the high adaptation of $\mathrm{LAB}$ to inhabit different plant niches of fruits and vegetables, which include flowers, grains, leaves, and/or grasses ( $\mathrm{Yu}$ et al., 2020). In addition, $\mathrm{Yu}$ et al. (2020) have reported the distinction between generalist- and specialist-LAB in plant ecosystems; the first ones occupy a wide range of habitats but with a variable degree of performance, whilst the specialists are present in a narrow range of habitats and they are highly adapted to them. The generalist-LAB have genomes of bigger size than the specialized once since they harbor a wide enzymatic machinery; an example of the first case is Lactiplantibacillus plantarum, widely isolated from plant and animal sources, meanwhile Fructilactobacillus sanfranciscensis is mainly associated with cereal (sourdough) fermentations. Another illustrative example of niche specialization is the case of LAB adapted to fructoserich niches, such as fruits, flowers, their fermented foods, and the intestines of certain insects; these bacteria have evolved to preferably use fructose instead of glucose (Filannino et al., 2019). This group of bacteria can be regarded as cell factories for the production of chemical and bioactives of interest (Figure 1A) due to their metabolic activity upon different substrates (Mazzoli et al., 2014; Sauer et al., 2017; Hatti-Kaul et al., 2018). There is not much information about the ecological distribution of LAB involved in the fermenting of plant waste, although it could be predicted that a similar degree of specialization is expected. Besides, the physical-chemical characteristics of the plant residues are different from those of the raw materials and their fermented food/feed products; thus, it could be expected that generalist LAB, or new specialized species, would be involved in plant-residue fermentations; thus, the waste matrix composition must also be taken into account in order for the selected LAB to optimize their performance during controlled fermentations. There is no doubt, that the fermentation of agro-food residues by $\mathrm{LAB}$, alone or in combination with other microorganisms, opens an avenue of opportunities for a sustainable circular economy.

\section{FERMENTATION PROCESSES EMPLOYING OTHER TYPES OF BACTERIA}

In addition to LAB-mediated fermentations, other fermentative bacteria have been applied to valorize vegetable by-products and other vegetable sources, including different Clostridium and Bacillus species. Most of these applications focused on the production of functional ingredients like lactic acid, Poly$\gamma$-glutamic acid, bioactive peptides to be reintegrated in the food chain, and other compounds like glycosidases or caproate of industrial interest (Table 1). Within the genus Bacillus, Bacillus coagulans, Bacillus amyloliquefaciens, Bacillus licheniformis and B. subtilis have been used, alone or together with other bacteria and/or fungi, to ferment products derived from rice, soy, oak, fruit, sorghum (Alexandri et al., 2019; Cole et al., 2019; Jiang et al., 2019; Mukherjee et al., 2019; Orts et al., 2019). Rice bran is an abundant by-product stream generated during rice processing that, after enzymatic hydrolysis, can be fermented by $B$. coagulans with the aim of producing highly pure (>99\%) L-lactic acid. Remarkably, the strain B. coagulans A107 was able to convert the sugars of the hydrolyzate to lactic acid with $90 \%$ yield, without additional nutritional requirements (Alexandri et al., 2019). On the other hand, soybean dregs, soybean meal and okara can also be fermented by Bacillus strains through different valorization ways. Soybean dregs can be transformed using poly- $\gamma$-glutamic acid producing stains of $B$. amyloliquefaciens in feed additives, resulting in fermentation products that could help to improve the growth indicators in animal experiments (Jiang et al., 2019). Soybean meal can be fermented by a mixed culture of $B$. coagulans and Lactobacillus johnsonii, yielding an improvement of antioxidant properties after fermentation (Mukherjee et al., 2019). Furthermore, B. licheniformis was able to ferment enzymatically hydrolyzed okara (a by-product of soy milk manufacturing) to produce soil biostimulants with higher $\beta$-glucosidase, phosphatase and dehydrogenase activities (Orts et al., 2019). These three examples show a variety of valorization processes mediated by Bacillus species that should be explored further to widen the range of applications of soyderived products.

Finally, it is worth highlighting the use of Clostridium species in the fermentation of fruit waste. In this regard, a successful fermentation strategy using Clostridium cellulovorans and Clostridium beijerinckii strains was designed to ferment mandarin orange waste. Normally, D-limonene included in citrus fruits inhibits yeast activity and makes ethanolic fermentation difficult. However, physiological concentration of D-limonene does not inhibit the growth of these two Clostridium strains. This allows the production of biofuels from this specific fruit waste, thanks to the isopropanol-butanol-ethanol fermentation ability of $C$. beijerinckii and the cellulosic biomass degrading ability of C. cellulovorans (Tomita et al., 2019).

\section{FERMENTATION PROCESSES EMPLOYING FUNGI AND YEASTS}

Fungi probably represent some of the first microorganisms that have been exploited in fermentation processes aimed at producing compounds of interest for medical, nutritional, and industrial applications (Kavanagh, 2005). Fungi produce a diverse array of extracellular enzymes, antibiotics and pigments, and, hence, growing them on vegetable wastes has long been used for their production. Some examples include lactic acid, functional carbohydrates, organic acids or carotenoids production to be reintegrated in the food chain as well as glycosidase and lipase enzymes (Table 1). Applications of fungal fermentation to enhance nutritional properties like fiber and protein content have been also reported (Table 1). For instance, diverse Aspergillus species have been 
A

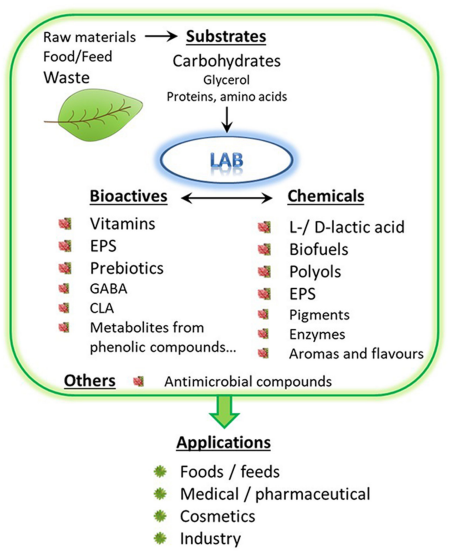

Scores plot - Neural network-based PCA

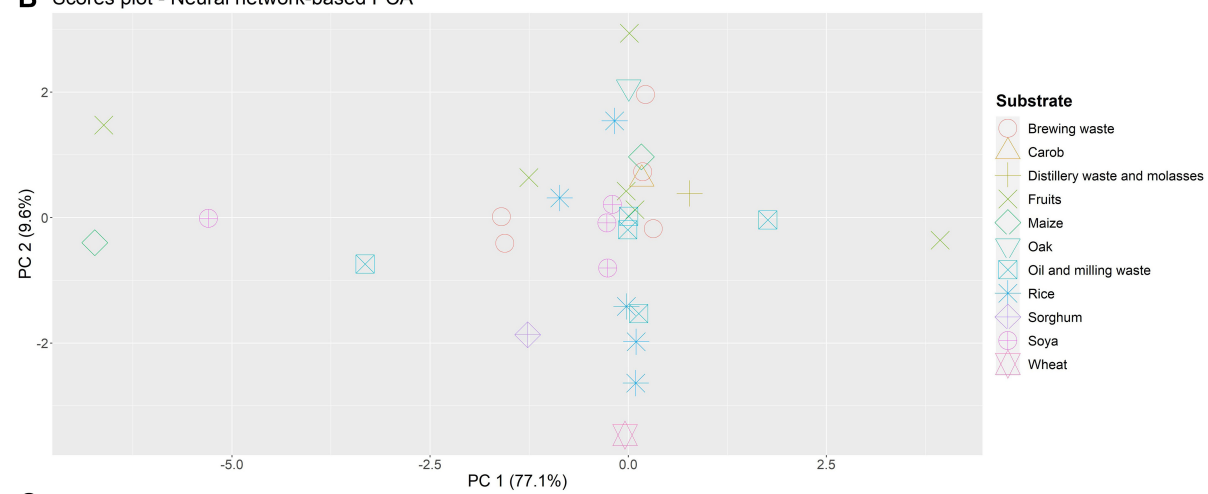

C scores plot - Neural network-based PCA

PC $1(77.1 \%)$
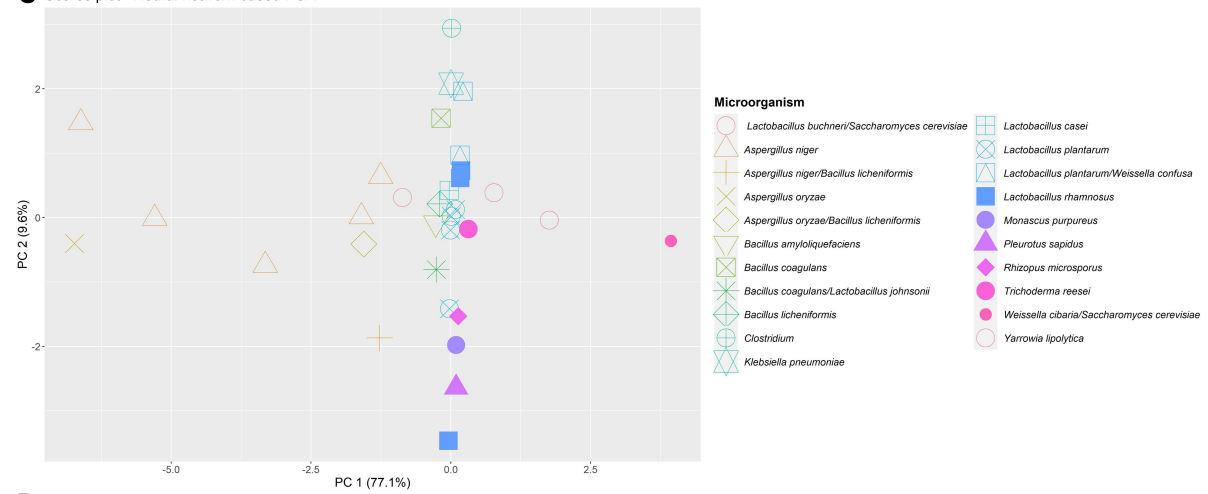

Scores plot - Neural network-based PCA

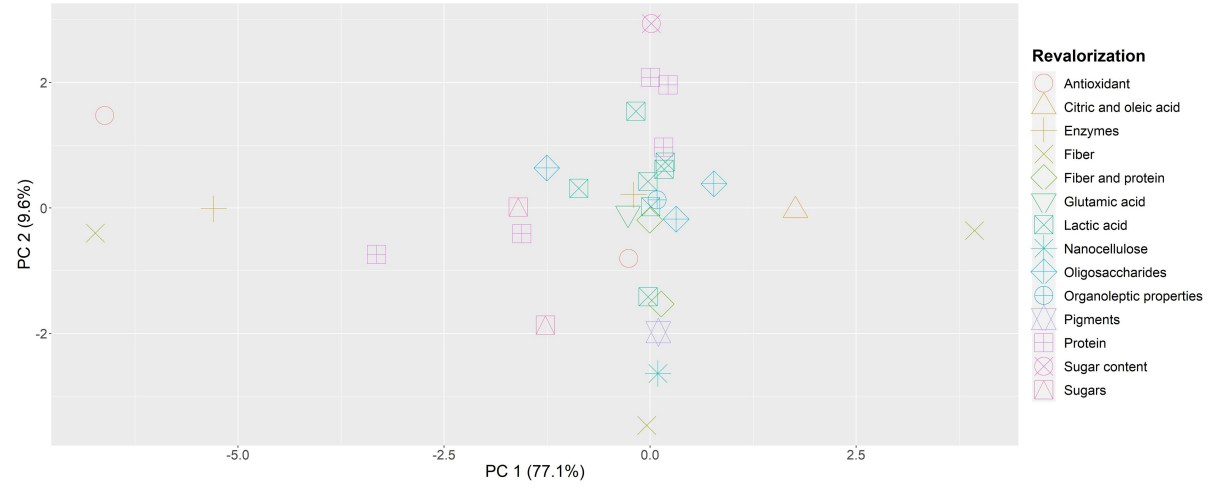

FIGURE 1 | Production of some chemical and/or bioactive compounds from plant-waste by the metabolic activity of lactic acid bacteria (LAB) (A) and distribution of fermentation data from studies found in the bibliography by artificial neural network-based principal component analysis (PCA). Differences according to substrate used (B), microorganisms inoculated (C) and valorization objective (D) and are shown. 
used to produce fungal enzymes with important technological applications, such as xylanases through brewer's spent grain fermentation (Beg et al., 2001); phytases through fermentation of triticale residues (Neira-Vielma et al., 2018), $\alpha$-amylases through fermentation of soya, wheat bran and other starchy residues (Mathew et al., 2016; Melnichuk et al., 2020), proteases through fermentation of soya bean and wheat bran (Novelli et al., 2015), or lipases through fermentation of lipid-rich agro-wastes such as olive pomaces (Oliveira et al., 2016). Of technological interest is also the production of natural pigments, such as the ones produced by the fermentation of rice pasta with Monascus purpureus, a process which also results in the production of an anti-hypercholesterolaemic agent (Jirasatid et al., 2019).

Indeed, fungal fermentation of agri-food wastes is used to produce a number of ingredients with added nutritional and health promoting attributes, either through favoring its release from the vegetable matrix, or through biotransformation of the compounds originally encountered in the residues. Thus, specific compounds produced through these processes are largely dependent on the raw material and the particular fungal species used. For instance, vegetable wastes are a rich source of nondigestible fibers and oligosaccharides which can confer improved technological (Cantatore et al., 2019), nutritional (Chebaibi et al., 2019), and functional properties when included in food preparations. Fungal fermentation of agri-food waste has been frequently used to produce prebiotic substrates, capable of beneficially modulating gut microbial populations (Gibson et al., 2011). In this regard, xylanolytic fungi, such as Aspergillus and Trichoderma species, produce arabinoxylo-oligosaccharides and xylose from cellulosic rich vegetables wastes, such as brewer's spent grain, rice husks, soybean hulls or grape pomaces (Amorim et al., 2019; Costa et al., 2019; Paz et al., 2019); and fructooligosaccharides have been produced through Aspergillus spp. fermentation of aguamiel (mead) from agave, sugar cane bagasse, or banana peel and/or leaves (Ganaie et al., 2017; Muñiz-Márquez et al., 2019), whereas isomaltulose, an alternative sweetener with prebiotic properties, is produced through the fermentation of cane molasses (Wang et al., 2019). The capability of generating food supplements with improved functional traits through fungal fermentation has been demonstrated in some works, for instance, supplementing mice diets with a sweet potato distillery waste fermented with Aspergillus oryzae, resulted in increased butyrate producers in the gut microbiota and lipid pool modulation (Kosakai et al., 2019).

Fungal fermentation of agri-food residues can also lead to the simultaneous production of multiple ingredients with added value. For instance, fermentation of olive-mill wastewaters by Yarrowia lipolytica to produce citric and oleic acid has been reported (Sarris et al., 2019), while a combination of Aspergillus, Pleurotus, and Hericium enabled the production of fractions rich in polyphenols, antioxidant activities, and fiber from the fermentation of a cooked-maize residue (Acosta-Estrada et al., 2019). Thus, the wide array of metabolic traits exhibited by fungal species offers a valuable scenario to design fermentation strategies capable of maximizing the sustainable production of added-value ingredients through vegetable residue fermentation.

\section{COMPARISON OF FERMENTATION PATTERNS FOR DIFFERENT APLICATIONS AND ECONOMIC FEASIBILITY}

Numerous studies dealing with the fermentation of fruit and vegetable by-products as an alternative way of valorization using different microorganisms have been reported in the bibliography. Table 1 provides a classification of substrates assayed in recent works and summarizes cultures and strains selected. Yields of functional ingredients isolated, and enhanced nutritional and technological properties of foods are also shown (reaction conditions for each process are provided in Supplementary Table 1). Moreover, Table 1 compares these fermentative processes which have been carried out in bioreactors to enzymatic hydrolysis using commercial enzyme preparations from bacteria and fungi (Cole et al., 2019; Costa et al., 2019; Marson et al., 2019; Paz et al., 2019).

To find common patterns in fermentation conditions optimized for each type of bioactive compound and nutritional/organoleptic properties, an artificial neural networkbased principal component analysis (PCA) is provided in Figures 1B-D. This type of model is especially suitable for data from biological experiments where the relationship between experimental variables is complex, and may help integrating heterogeneous data reported by previous authors to give an overall view of fermentation applications designed for a wide variety of substrates and microorganisms. This model combines a conventional PCA model with artificial neural networks in order to reconstruct experimental data describing as much variance as possible (Stacklies et al., 2007). Artificial neural networks are powerful pattern-recognition algorithms formed by an input layer (i.e., principal components from PCA), an output layer (i.e., reconstructed fermentation conditions) and several neurons or nodes organized in a hidden layer, connected through mathematical functions. In this case, the model was built with six neurons in the hidden layer and was able to explain the high proportion of variance, as shown in Figures 1B-D. Differences and similarities in fermentation processes according to industrial by-products used as substrates (Figure 1B), microbial cultures (Figure 1C), and valorization objectives (Figure 1D) can easily be elucidated through graphical representation.

\section{Reaction Conditions According to the Substrate}

Some differences in fermentative conditions according to each type of fruit and vegetable by-product assayed were observed (Figure 1B). Soya by-products (Jiang et al., 2019; Mukherjee et al., 2019; Orts et al., 2019; Taddia et al., 2019) were mostly subjected to solid-state fermentation at $30-47^{\circ} \mathrm{C}$ using Aspergillus niger and Bacillus species or yeasts employing lower temperatures (20-28 $\left.{ }^{\circ} \mathrm{C}\right)$ (Papadaki et al., 2019; Chua and Liu, 2020). Barley bran and brewing waste (Amorim et al., 2019; Marson et al., 2019; Outeiriño et al., 2019; Paz et al., 2019; Pejin et al., 2019; Pontonio et al., 2020) was mostly inoculated with Aspergillus, 
Trichoderma, and LAB species. Rice bran and husk fermentations (Alexandri et al., 2019; Jirasatid et al., 2019; Montipó et al., 2019; Postemsky et al., 2019; Saman et al., 2019) carried out at $\mathrm{pH}$ 6.0-6.9 were mainly aimed at lactic acid production compared to other substrates. A great variability was observed in fermentation conditions of fruit by-products depending on the application. Some applications report the use of LAB at different times 72240 h (Ricci et al., 2019b; Cheng et al., 2020) and no clear patterns could be inferred, although these samples were also differentiated from the rest of substrates.

\section{Reaction Conditions According to the Microorganism Used}

With regard to microbial cultures assayed (Figure 1C), fermentations using Clostridium, B. subtilis, combination of B. coagulans and L. johnsonii, Pleurotus sapidus, A. niger, and $A$. oryzae showed the highest differences in their reactive conditions. Of all the studies compared, fermentations with mixed cultures of $C$. beijerinckii NCIMB8052 and C. cellulovorans $743 \mathrm{~B}$ to reduce sugar content $(-85 \%)$ in citrus by-products (Tomita et al., 2019) were carried out with the lowest substrate concentration (1\%) at prolonged reaction times (384 h). Similarly, vegetable wastes predominantly fermented by Clostridium kluyveri to obtain caproate were carried out at the highest reaction times in a two-step process comprising $80 \mathrm{~h}$ and 70 days (Yu et al., 2019). In this sense, fermentations of soya by-products using B. subtilis (Ruan et al., 2020) and combination of $B$. coagulans and L. johnsonii (Mukherjee et al., 2019) were performed at prolonged times (>140 h) and high initial $\mathrm{pH}(6.7-7.0)$. These processes where differentiated from applications using both Weissella cibaria PEP23F and Saccharomyces cerevisiae AN6Y19 to increase fiber content $(+40 \%)$ in apple residues (Cantatore et al., 2019) that exhibited low initial $\mathrm{pH}$ (4.0), high substrate concentrations and shorter fermentation times $(48 \mathrm{~h})$.

On the other hand, some fermentation conditions of rice husks by $P$. sapidus MkP6 to release nanocellulose $(670 \mathrm{mg} / \mathrm{g}$ ) (Postemsky et al., 2019), were similar to those of Lacticaseibacillus rhamnosus 1473 , to increase arabinoxylan solubility (+200\%) from wheat bran (Spaggiari et al., 2020). These two processes were solid-state fermentations where the humidity of substrates was adjusted to $60-75 \%$, with an initial $\mathrm{pH}$ of 6.0-6.5. However, these processes were conducted at different times (20 and $48 \mathrm{~h}$ ). In contrast, $A$. oryzae fermentations of maize-milling waste in co-culture with Pleurotus ostreatus and Hericium erinaceus to increase fiber content $(+45 \%)$ (Acosta-Estrada et al., 2019) were performed under similar conditions to those processes using A. niger. Specifically, A. niger strains NRRL3, GH1 and enzymes isolated from CECT 2700 and 3T5B8 were used to treat mango, grape, soya and brewing by-products, and olive cake, leading to a high antioxidant activity (90\%), release of xylo-oligosaccharides (887 mg/g), enzymes (soya $555 \mathrm{U} / \mathrm{mL}$ ), xylose $(6 \mathrm{~g} / \mathrm{L})$, and an increase in protein content (+94\%) (Chebaibi et al., 2019; Costa et al., 2019; Paz et al., 2019; Taddia et al., 2019; Torres-León et al., 2019). Most of these processes were solid state-fermentations. In general, the use of fungi is preferred to isolate glycosidases and functional oligosaccharides while applications of bacteria are focused on other types of compounds such as lactic acid.

\section{Reaction Conditions According to the Valorization Objective}

Finally, differences in fermentation conditions can also be observed depending on the valorization objective (Figure 1D). Those bioprocesses aimed at releasing oligosaccharides (Amorim et al., 2019; Costa et al., 2019; Muñiz-Márquez et al., 2019; Wang et al., 2019) were characterized by low substrate concentrations (5-35\%) inoculated with fungi from Aspergillus, Trichoderma, and Yarrowia genus at temperatures of $30-40^{\circ} \mathrm{C}$. Fermentations designed to produce lactic acid (Alexandri et al., 2019; Bahry et al., 2019; do Carmo Brito et al., 2019; Goto et al., 2019; Mladenović et al., 2019a,b; Montipó et al., 2019; Pejin et al., 2019; Ricci et al., 2019b; Saman et al., 2019) where LAB species were mostly inoculated, were performed with low raw material concentrations (7-25\%) at $\mathrm{pH}$ 6.0-6.6. Moreover, fermentative applications to increase protein content of matrices (Aruna, 2019; Chebaibi et al., 2019; Marson et al., 2019) were characterized by the use of molds from Aspergillus and Trichoderma genus. Interestingly, similar reaction conditions were reported to obtain lipases, nanocellulose, and pigments (Jirasatid et al., 2019; Postemsky et al., 2019; Tišma et al., 2019). These processes were solid-state fermentations using fungi at high concentrations of rice and milling by-products ( $>39 \%)$ and an initial $\mathrm{pH}$ of 6.0-7.0.

\section{Economic Feasibility Consideration}

Previous studies demonstrate the economic feasibility of vegetable by-product fermentation to obtain high added-value ingredients (Lam et al., 2014; Manandhar and Shah, 2020). Fermentation of vegetable by-products using bacteria, fungi, and yeast showed a return on investment and an internal rate of return above $10 \%$, and a breakeven of the capital investment of approximately 7 years (Lam et al., 2014; Serna-Loaiza et al., 2019; Manandhar and Shah, 2020). To consider the economic feasibility of fermentation applications discussed in this minireview, the selling price for functional ingredients presented in Table $\mathbf{1}$ was collected and compared to fermentation time and product yield (Supplementary Table 2). It should be noted that only those articles reporting fermentation yields and complete process conditions were considered for comparative purposes. In this sense, the applications that showed the highest ratio between selling price, yield, and fermentation time were the production of xylo-oligosaccharides, poly- $\gamma$-glutamic, acid and arabinoxylooligosaccharides from grape pomace flour, soybean dregs, and brewer's spent grain, respectively. This fact is attributed to the high selling price of these ingredients. In contrast, the processes that showed the lowest ratio were the production of carotenoids, isomaltulose and glycosidases from soya by-products and cane molasses, due to the prolonged fermentation times needed and/or lower product price compared to other functional ingredients. It should be noted that no major differences in these ratios were found depending on the microorganism used (bacteria, fungi, or yeast). In summary, production of potentially prebiotic oligosaccharides and biopolymers from vegetable by-products 
may be highly profitable strategies to valorize vegetable byproducts, regardless the microorganism selected. It should be taken into account that the comparison above discussed provides tentative information about the profitability of these applications. However, a detailed cost analysis of each process is needed to further ensure feasibility before their implementation in the industry.

A wide range of bacterial and fungal species to treat several agricultural wastes have been reported in literature. Advances in microbial culture analysis and its integration with omics techniques will boost current understanding of mixed culture fermentation (De Groof et al., 2019) in order to design efficient fermentative processes for specific applications. The present work provides a classification of bacteria and fungi species and strains currently used for bioconversion of fruit and vegetable by-products, and a comparison of fermentative conditions needed to isolate specific functional compounds, or to achieve certain nutritional goals. Most fermentation applications compared in this review could be economically feasible considering the high product yields reported. Among fermentation processes discussed, the most profitable valorization strategies may be the obtainment of functional oligosaccharides and poly- $\gamma$-glutamic acid from soya by-products and molasses using A. niger, A. aculeatus, T. reesei, or B. amyloliquefaciens using specific substrate concentration, temperature, and reaction time conditions. On the other hand, enzyme obtainment from these by-products might not be profitable due to the prolonged fermentation times needed. The information summarized may help in the production of

\section{REFERENCES}

Acosta-Estrada, B. A., Villela-Castrejón, J., Perez-Carrillo, E., Gómez-Sánchez, C. E., and Gutiérrez-Uribe, J. A. (2019). Effects of solid-state fungi fermentation on phenolic content, antioxidant properties and fiber composition of lime cooked maize by-product (nejayote). J. Cereal Sci. 90:102837. doi: 10.1016/j.jcs. 2019.102837

Alexandri, M., Neu, A. K., Schneider, R., López-Gómez, J. P., and Venus, J. (2019). Evaluation of various Bacillus coagulans isolates for the production of high purity L-lactic acid using defatted rice bran hydrolysates. Int. J. Food Sci. Tech. 54, 1321-1329. doi: 10.1111/ijfs. 14086

Amorim, C., Silvério, S. C., and Rodrigues, L. R. (2019). One-step process for producing prebiotic arabino-xylooligosaccharides from brewer's spent grain employing Trichoderma species. Food Chem. 270, 86-94. doi: 10.1016/j. foodchem.2018.07.080

Aruna, T. E. (2019). Production of value-added product from pineapple peels using solid state fermentation. Innov. Food Sci. Emerg. Technol. 57:102193. doi: $10.1016 /$ j.ifset.2019.102193

Avila, C. L. S., and Carvalho, B. F. (2020). Silage fermentation-updates focusing on the performance of micro-organisms. J. Appl. Microbiol. 128, 966-984. doi: 10.1111/jam.14450

Azam, M. T., and Ahmad, A. (2019). "Date palm waste: an efficient source for production of glucose and lactic acid" in Sustainable Agriculture Reviews 34, eds Naushad, M., and Lichtfouse, E. (Germany: Springer), 155-178. doi: 10.1007/ 978-3-030-11345-2_8

Bachtarzi, N., Kharroub, K., and Ruas-Madiedo, P. (2019). Algerian dairy products and their application for skim-milk fermentations. LWT 107, 117-124. doi: 10.1016/j.lwt.2019.03.005

Bahry, H., Abdalla, R., Pons, A., Taha, S., and Vial, C. (2019). Optimization of lactic acid production using immobilized Lactobacillus rhamnosus and carob bioactive ingredients for novel food formulation as well as in the development of low-cost bioprocesses leading to a transition toward a bioeconomy model.

\section{AUTHOR CONTRIBUTIONS}

All authors listed have made a substantial, direct and intellectual contribution to the work, and approved it for publication.

\section{FUNDING}

The research performed in the MicroHealth research group was funded by the European Union's Horizon 2020 Research and Innovation Program under grant agreement No. 818368 (MASTER), the project IDI/2018/000236 (funded by PCTI Gobierno del Principado de Asturias/FEDER, UE), and the grant RTI2018-095021-J-I00 (funded by MCIU/AEI/FEDER, UE). CS acknowledges his Postdoctoral research contract funded by the Instituto de Investigación Sanitaria del Principado de Asturias (ISPA).

\section{SUPPLEMENTARY MATERIAL}

The Supplementary Material for this article can be found online at: https://www.frontiersin.org/articles/10.3389/fmicb. 2020.581997/full\#supplementary-material

pod waste from the Lebanese food industry. J. Biotechnol. 306, 81-88. doi: 10.1016/j.jbiotec.2019.09.017

Bedoić, R., Ćosić, B., and Duić, N. (2019). Technical potential and geographic distribution of agricultural residues, co-products and by-products in the European Union. Sci. Total Environ. 686, 568-579. doi: 10.1016/j.scitotenv. 2019.05.219

Beg, Q. K., Kapoor, M., Mahajan, L., and Hoondal, G. S. (2001). Microbial xylanases and their industrial applications: a review. Appl. Microbiol. Biotechnol. 56, 326-338. doi: 10.1007/s002530100704

Bron, P. A., Marcelli, B., Mulder, J., van der Els, S., Morawska, L. P., Kuipers, O. P., et al. (2019). Renaissance of traditional DNA transfer strategies for improvement of industrial lactic acid bacteria. Curr. Opin. Biotech. 56, 61-68. doi: 10.1016/j.copbio.2018.09.004

Brückner-Gühmann, M., Banovic, M., and Drusch, S. (2019). Towards an increased plant protein intake: rheological properties, sensory perception and consumer acceptability of lactic acid fermented, oat-based gels. Food Hydrocoll. 96, 201208. doi: 10.1016/j.foodhyd.2019.05.016

Burke, D. G., Cotter, P. D., Ross, R. P., and Hill, C. (2013). "Microbial production of bacteriocins for use in foods," in Microbial Production of Food Ingredients, Enzymes and Nutraceuticals, eds B. McNeil, D. Archer, I. Giavasis, and L. Harvey (Sawston: Woodhead Publishing), 353-384. doi: 10.1533/9780857093547.2.353

Cantatore, V., Filannino, P., Giuseppe, G., De Pasquale, I., Pan, S., Gobbetti, M., et al. (2019). Lactic acid fermentation to re-cycle apple by-products for wheat bread fortification. Front. Microbiol. 10:2574. doi: 10.3389/fmicb.2019.02574

Chebaibi, S., Grandchamp, M. L., Burgé, G., Clément, T., Allais, F., and Laziri, F. (2019). Improvement of protein content and decrease of anti-nutritional factors in olive cake by solid-state fermentation: a way to valorize this industrial byproduct in animal feed. J. Biosci. Bioeng. 128, 384-390. doi: 10.1016/j.jbiosc. 2019.03.010

Cheng, Y., Wu, T., Chu, X., Tang, S., Cao, W., Liang, F., et al. (2020). Fermented blueberry pomace with antioxidant properties improves fecal microbiota 
community structure and short chain fatty acids production in an in vitro mode. LWT 125:109260. doi: 10.1016/j.lwt.2020.109260

Chua, J. Y., and Liu, S. Q. (2020). Effect of single amino acid addition on growth kinetics and flavor modulation by Torulaspora delbrueckii in soy (tofu) whey alcoholic beverage fermentation. Food Res. Int. 135:109283. doi: 10.1016/j. foodres.2020.109283

Cole, M. R., Eggleston, G., Gaines, D. K., and Heckemeyer, M. (2019). Development of an enzyme cocktail to bioconvert untapped starch in sweet sorghum processing by-products: part I. Ind. Crop Prod. 133, 142-150. doi: 10.1016/j. indcrop.2019.03.012

Costa, J. R., Tonon, R. V., Gottschalk, L. M., Santiago, M. C. D. A., Mellinger-Silva, C., Pastrana, L., et al. (2019). Enzymatic production of xylooligosaccharides from Brazilian Syrah grape pomace flour: a green alternative to conventional methods for adding value to agricultural by-products. J. Sci. Food Agric. 99, 1250-1257. doi: 10.1002/jsfa.9297

De Groof, V., Coma, M., Arnot, T., Leak, D. J., and Lanham, A. B. (2019). Medium chain carboxylic acids from complex organic feedstocks by mixed culture fermentation. Molecules 24:398. doi: 10.3390/molecules24030398

do Carmo Brito, B. D. N., Chiste, R. C., Lopes, A. S., Glória, M. B. A., and da Silva Pena, R. (2019). Influence of spontaneous fermentation of manipueira on bioactive amine and carotenoid profiles during tucupi production. Food Res. Int. 120, 209-216. doi: 10.1016/j.foodres.2019.02.040

Filannino, P., Di Cagno, R., and Gobbetti, M. (2018). Metabolic and functional paths of lactic acid bacteria in plant foods: get out of the labyrinth. Curr. Opin. Biotech. 49, 64-72. doi: 10.1016/j.copbio.2017.07.016

Filannino, P., Di Cagno, R., Tlais, A. Z. A., Cantatore, V., and Gobbetti, M. (2019). Fructose-rich niches traced the evolution of lactic acid bacteria toward fructophilic species. Crit. Rev. Microbiol. 45, 65-81. doi: 10.1080/1040841X. 2018.1543649

Ganaie, M. A., Soni, H., Naikoo, G. A., Oliveira, L. T. S., Rawat, H. K., Mehta, P. K., et al. (2017). Screening of low cost agricultural wastes to maximize the fructosyltransferase production and its applicability in generation of fructooligosaccharides by solid state fermentation. Int. Biodeter. Biodegr. 118, 19-26. doi: 10.1016/j.ibiod.2017.01.006

Gibbons, J. G., and Rinker, D. C. (2015). The genomics of microbial domestication in the fermented food environment. Curr. Opin. Genet. Dev. 35, 1-8. doi: 10.1016/j.gde.2015.07.003

Gibson, G., Scott, K., Rastall, R., Tuohy, K., Hotchkiss, A., DubertFerrandon, A., et al. (2011). Dietary prebiotics: current status and new definition. Food Sci. Technol. Bull. 7, 1-19. doi: 10.1616/1476-2137. 15880

Gobbetti, M., De Angelis, M., Di Cagno, R., Calasso, M., Archetti, G., and Rizzello, C. G. (2019). Novel insights on the functional/nutritional features of the sourdough fermentation. Int. J. Food Microbiol. 302, 103-113. doi: 10.1016/j. ijfoodmicro.2018.05.018

Goto, M., Kuda, T., Shikano, A., Charrouf, Z., Yamauchi, K., Yokozawa, M., et al. (2019). Induction of superoxide anion radical-scavenging capacity in an argan press cake-suspension by fermentation using Lactobacillus plantarum Argan-L1. LWT 100, 56-61. doi: 10.1016/j.lwt.2018.10.033

Hatti-Kaul, R., Chen, L., Dishisha, T., and El Enshasy, H. (2018). Lactic acid bacteria: from starter cultures to producers of chemicals. FEMS Microbiol. Lett. 365:fny213. doi: 10.1093/femsle/fny213

Hidalgo-Cantabrana, C., O'Flaherty, S., and Barrangou, R. (2017). CRISPR-based engineering of next-generation lactic acid bacteria. Curr. Opin. Microbiol. 37, 79-87. doi: 10.1016/j.mib.2017.05.015

Jiang, K., Tang, B., Wang, Q., Xu, Z., Sun, L., Ma, J., et al. (2019). The bio-processing of soybean dregs by solid state fermentation using a poly $\gamma$-glutamic acid producing strain and its effect as feed additive. Bioresour. Technol. 291:121841. doi: 10.1016/j.biortech.2019.121841

Jirasatid, S., Limroongreungrat, K., and Nopharatana, M. (2019). Monacolin K, pigments and citrinin of rice pasta by-products fermented by Monascus purpureus. Int. Food Res. J. 26, 12791284.

Kavanagh, K. (2005). "Fungal fermentation systems and products," in Fungi: Biology and Applications, ed. K. Kavanagh (Hoboken, NJ: John Wiley \& Sons), 125. doi: 10.1002/9781119976950.ch5

Kimoto-Nira, H., Moriya, N., Nogata, Y., Sekiyama, Y., and Toguchi, Y. (2019). Fermentation of Shiikuwasha (Citrus depressa Hayata) pomace by lactic acid bacteria to generate new functional materials. Int. J. Food Sci. Tech. 54, 688-695. doi: $10.1111 /$ ijfs. 13980

Kosakai, T., Kato, H., Sho, C., Kawano, K., Iwai, K. I., Takase, Y., et al. (2019). Dietary fermented products using koji mold and sweet potato-shochu distillery by-product promotes hepatic and serum cholesterol levels and modulates gut microbiota in mice fed a high-cholesterol diet. PeerJ 7:e7671. doi: 10.7717/peerj. 7671

Lam, K. F., Leung, C. C. J., Lei, H. M., and Lin, C. S. K. (2014). Economic feasibility of a pilot-scale fermentative succinic acid production from bakery wastes. Food Bioprod. Process. 92, 282-290. doi: 10.1016/j.fbp.2013.09.001

Li, Q., and Gänzle, M. G. (2020). Host-adapted lactobacilli in food fermentations: impact of metabolic traits of host adapted lactobacilli on food quality and human health. Curr. Opin. Food Sci. 31, 71-80. doi: 10.1016/j.cofs.2020.02.002

Lu, Z., Wang, J., Gao, R., Ye, F., and Zhao, G. (2019). Sustainable valorisation of tomato pomace: a comprehensive review. Trends Food Sci. Tech. 86, 172-187. doi: 10.1016/j.tifs.2019.02.020

Lücke, F. K., Fritz, V., Tannhäuser, K., and Arya, A. (2019). Controlled fermentation of rapeseed presscake by Rhizopus, and its effect on some components with relevance to human nutrition. Food Res. Int. 120, 726-732. doi: 10.1016/j.foodres.2018.11.031

Manandhar, A., and Shah, A. (2020). Techno-economic analysis of bio-based lactic acid production utilizing corn grain as feedstock. Processes 8:199. doi: 10.3390/ pr8020199

Marson, G. V., da CostaMachado, M. T., de Castro, R. J. S., and Hubinger, M. D. (2019). Sequential hydrolysis of spent brewer's yeast improved its physicochemical characteristics and antioxidant properties: a strategy to transform waste into added-value biomolecules. Process Biochem. 84, 91-102. doi: 10.1016/ j.procbio.2019.06.018

Mathew, J. J., Vazhacharical, P. J., Sajeskhumar, N. K., and Ashokan, A. (2016). Amylase production by Aspergillus niger through submerged fermentation using starchy food byproducts as substrate. Int. J. Herb. Med. 4, 34-40.

Mazzoli, R., Bosco, F., Mizrahi, I., Bayer, E. A., and Pessione, E. (2014). Towards lactic acid bacteria-based biorefineries. Biotechnol. Adv. 32, 1216-1236. doi: 10.1016/j.biotechadv.2014.07.005

Melnichuk, N., Braia, M. J., Anselmi, P. A., Meini, M. R., and Romanini, D. (2020). Valorization of two agroindustrial wastes to produce alpha-amylase enzyme from Aspergillus oryzae by solid-state fermentation. Waste Manag. 106, 155-161. doi: 10.1016/j.wasman.2020.03.025

Mladenović, D., Djukić-Vuković, A., Stanković, M., Milašinović-Šeremešić, M., Radosavljević, M., Pejin, J., et al. (2019a). Bioprocessing of agro-industrial residues into lactic acid and probiotic enriched livestock feed. J. Sci. Food Agric. 99, 5293-5302. doi: 10.1002/jsfa.9759

Mladenović, D., Pejin, J., Kocić-Tanackov, S., Djukić-Vuković, A., and Mojović, L. (2019b). Enhanced lactic acid production by adaptive evolution of Lactobacillus paracasei on agro-industrial substrate. Appl. Biochem. Biotech. 187, 753-769. doi: 10.1007/s12010-018-2852-x

Montipó, S., Ballesteros, I., Fontana, R. C., Liu, S., Ballesteros, M., Martins, A. F., et al. (2019). Bioprocessing of rice husk into monosaccharides and the fermentative production of bioethanol and lactate. Cellulose 26, 7309-7322. doi: 10.1007/s10570-019-02571-1

Mukherjee, R., Chakraborty, R., and Dutta, A. (2019). Comparison of optimization approaches (response surface methodology and artificial neural networkgenetic algorithm) for a novel mixed culture approach in soybean meal fermentation. J. Food Process Eng. 42:e13124. doi: 10.1111/jfpe.13124

Muñiz-Márquez, D. B., Teixeira, J. A., Mussatto, S. I., Contreras-Esquivel, J. C., Rodríguez-Herrera, R., and Aguilar, C. N. (2019). Fructo-oligosaccharides (FOS) production by fungal submerged culture using aguamiel as a low-cost by-product. LWT 102, 75-79. doi: 10.1016/j.lwt.2018.12.020

Neira-Vielma, A. A., Aguilar, C. N., Ilyina, A., Contreras-Esquivel, J. C., Carneiroda-Cunha, M. G., Michelena-Álvarez, G., et al. (2018). Purification and biochemical characterization of an Aspergillus niger phytase produced by solidstate fermentation using triticale residues as substrate. Biotechnol. Rep. 17, 49-54. doi: 10.1016/j.btre.2017.12.004

Novelli, P. K., Barros, M. M., and Fleuri, L. F. (2015). Novel inexpensive fungi proteases: production by solid state fermentation and characterization. Food Chem. 198, 119-124. doi: 10.1016/j.foodchem.2015.11.089

Oliveira, F., Moreira, C., Salgado, J. M., Abrunhosa, L., Venâncio, A., and Belo, I. (2016). Olive pomace valorization by Aspergiullus species: lipase production 
using solid-state fermentation. J. Sci. Food Agric. 96, 3583-3589. doi: 10.1002/ jsfa.75544

Orts, Á, Tejada, M., Parrado, J., Paneque, P., García, C., Hernández, T., et al. (2019). Production of biostimulants from okara through enzymatic hydrolysis and fermentation with Bacillus licheniformis: comparative effect on soil biological properties. Environ. Technol. 40, 2073-2084. doi: 10.1080/09593330.2018. 1436596

Outeiriño, D., Costa-Trigo, I., de Souza Oliveira, R. P., Guerra, N. P., and Domínguez, J. M. (2019). A novel approach to the biorefinery of brewery spent grain. Process Biochem. 85, 135-142. doi: 10.1016/j.procbio.2019.06.007

Papadaki, A., Kopsahelis, N., Mallouchos, A., Mandala, I., and Koutinas, A. A. (2019). Bioprocess development for the production of novel oleogels from soybean and microbial oils. Food Res. Int. 126:108684. doi: 10.1016/j.foodres. 2019.108684

Paz, A., Outeiriño, D., Guerra, N. P., and Domínguez, J. M. (2019). Enzymatic hydrolysis of brewer's spent grain to obtain fermentable sugars. Bioresour. Technol. 275, 402-409. doi: 10.1016/j.biortech.2018.12.082

Pejin, J., Radosavljević, M., Kocić-Tanackov, S., Marković, R., Djukić-Vuković, A., and Mojović, L. (2019). Use of spent brewer's yeast in L-(+) lactic acid fermentation. J. Inst. Brew. 125, 357-363. doi: 10.1002/jib.572

Petrova, P., and Petro, K. (2020). Lactic acid fermentation of cereals and pseudocereals: ancient nutritional biotechnologies with modern applications. Nutrients 12:1118. doi: 10.3390/nu12041118

Pontonio, E., Dingeo, C., Di Cagno, R., Blandino, M., Gobbetti, M., and Rizzello, C. G. (2020). Brans from hull-less barley, emmer and pigmented wheat varieties: from by-products to bread nutritional improvers using selected lactic acid bacteria and xylanase. Int. J. Food Microbiol. 313:108384. doi: 10.1016/j. ijfoodmicro.2019.108384

Pontonio, E., Dingeo, C., Gobbetti, M., and Rizzello, C. G. (2019). Maize milling byproducts: from food wastes to functional ingredients through lactic acid bacteria fermentation. Front. Microbiol. 10:561. doi: 10.3389/fmicb.2019.00561

Postemsky, P. D., Bidegain, M. A., Lluberas, G., Lopretti, M. I., Bonifacino, S., Landache, M. I., et al. (2019). Biorefining via solid-state fermentation of rice and sunflower by-products employing novel monosporic strains from Pleurotus sapidus. Bioresour. Technol. 289:121692. doi: 10.1016/j.biortech.2019.121692

Ricci, A., Bernini, V., Maoloni, A., Cirlini, M., Galaverna, G., Neviani, E., et al. (2019a). Vegetable by-product lacto-fermentation as a new source of antimicrobial compounds. Microorganisms 7:607. doi: 10.3390/ microorganisms7120607

Ricci, A., Diaz, A. B., Caro, I., Bernini, V., Galaverna, G., Lazzi, C., et al. (2019b) Orange peels: from by-product to resource through lactic acid fermentation. J. Sci. Food Agric. 99, 6761-6767. doi: 10.1002/jsfa.9958

Ruan, S., Luo, J., Li, Y., Wang, Y., Huang, S., Lu, F., et al. (2020). Ultrasoundassisted liquid-state fermentation of soybean meal with Bacillus subtilis: effects on peptides content, ACE inhibitory activity and biomass. Process Biochem. 91, 73-82. doi: 10.1016/j.procbio.2019.11.035

Saman, P., Fuciños, P., Vázquez, J. A., and Pandiella, S. S. (2019). By-products of the rice processing obtained by controlled debranning as substrates for the production of probiotic bacteria. Innov. Food Sci. Emerg. Technol. 51, 167-176. doi: 10.1016/j.ifset.2018.05.009

Sarris, D., Rapti, A., Papafotis, N., Koutinas, A. A., and Papanikolaou, S. (2019). Production of added-value chemical compounds through bioconversions of olive-mill wastewaters blended with crude glycerol by a Yarrowia lipolytica strain. Molecules 24:222. doi: 10.3390/molecules24020222

Satari, B., and Karimi, K. (2018). Citrus processing wastes: environmental impacts, recent advances, and future perspectives in total valorization. Resour. Conserv. Recy. 129, 153-167. doi: 10.1016/j.resconrec.2017.10.032

Sauer, M., Russmayer, H., Grabherr, R., Peterbauer, C. K., and Marx, H. (2017). The efficient clade: lactic acid bacteria for industrial chemical production. Trends Biotechnol. 35, 756-769. doi: 10.1016/j.tibtech.2017.05.002

Schettino, R., Pontonio, E., and Rizzello, C. G. (2019). Use of fermented hemp, chickpea and milling by-products to improve the nutritional value of semolina pasta. Foods 8:604. doi: 10.3390/foods8120604

Serna-Loaiza, S., García-Velásquez, C. A., and Cardona, C. A. (2019). Strategy for the selection of the minimum processing scale for the economic feasibility of biorefineries. Biofuel Bioprod. Biorefin. 13, 107-119. doi: 10.1002/bbb. 1941

Spaggiari, M., Ricci, A., Calani, L., Bresciani, L., Neviani, E., Dall'Asta, C., et al. (2020). Solid state lactic acid fermentation: a strategy to improve wheat bran functionality. LWT 118:108668. doi: 10.1016/j.lwt.2019.108668

Stacklies, W., Redestig, H., Scholz, M., Walther, D., and Selbig, J. (2007). pcaMethods-a bioconductor package providing PCA methods for incomplete data. Bioinformatics 23, 1164-1167. doi: 10.1093/bioinformatics/btm069

Taddia, A., Boggione, M. J., and Tubio, G. (2019). Screening of different agroindustrial by-products for industrial enzymes production by fermentation processes. Int. J. Food Sci. Tech. 54, 1027-1035. doi: 10.1111/ijfs.13915

Tišma, M., Tadić, T., Budžaki, S., Ostojèić, M., Šalić, A., Zelić, B., et al. (2019). Lipase production by solid-state cultivation of Thermomyces lanuginosus on by-products from cold-pressing oil production. Processes 7:465. doi: 10.3390/ pr7070465

Tomita, H., Okazaki, F., and Tamaru, Y. (2019). Direct IBE fermentation from mandarin orange wastes by combination of Clostridium cellulovorans and Clostridium beijerinckii. AMB Express 9:1. doi: 10.1186/s13568-018-0728-7

Torres, S., Verón, H., Contreras, L., and Isla, M. I. (2020). An overview of plantautochthonous microorganisms and fermented vegetable foods. Food Sci. Hum. Well. 9, 112-123. doi: 10.1016/j.fshw.2020.02.006

Torres-León, C., Ramírez-Guzmán, N., Ascacio-Valdés, J., Serna-Cock, L., dos Santos Correia, M. T., Contreras-Esquivel, J. C., et al. (2019). Solid-state fermentation with Aspergillus niger to enhance the phenolic contents and antioxidative activity of Mexican mango seed: a promising source of natural antioxidants. LWT 112:108236. doi: 10.1016/j.lwt.2019.06.003

Valenti, F., Porto, S. M., Selvaggi, R., and Pecorino, B. (2020). Co-digestion of by-products and agricultural residues: a bioeconomy perspective for a Mediterranean feedstock mixture. Sci. Total Environ. 700:134440. doi: 10.1016/ j.scitotenv.2019.134440

Vásquez, Z. S., de Carvalho Neto, D. P., Pereira, G. V., Vandenberghe, L. P., de Oliveira, P. Z., Tiburcio, P. B., et al. (2019). Biotechnological approaches for cocoa waste management: a review. Waste Manag. 90, 72-83. doi: 10.1016/j. wasman.2019.04.030

Verni, M., Rizzello, C. G., and Coda, R. (2019). Fermentation biotechnology applied to cereal industry by-products: nutritional and functional insights. Front. Nutr. 6:42. doi: 10.3389/fnut.2019.00042

Wang, Z. P., Wang, Q. Q., Liu, S., Liu, X. F., Yu, X. J., and Jiang, Y. L. (2019). Efficient conversion of cane molasses towards high-purity isomaltulose and cellular lipid using an engineered Yarrowia lipolytica strain in fed-batch fermentation. Molecules 24:1228. doi: 10.3390/molecules 2407 1228

Wuyts, S., Van Beeck, W., Allonsius, C. N., van den Broek, M. F. L., and Lebeer, S. (2020). Applications of plant-based fermented foods and their microbes. Curr. Opin. Biotech. 61, 45-52. doi: 10.1016/j.copbio.2019. 09.023

Yu, A. O., Leveau, J. H. J., and Marco, M. L. (2020). Abundance, diversity and plantspecific adaptations of plant-associated lactic acid bacteria. Environ. Microbiol. Rep. 12, 16-29. doi: 10.1111/1758-2229.12794

Yu, J., Huang, Z., Wu, P., Zhao, M., Miao, H., Liu, C., et al. (2019). Performance and microbial characterization of two-stage caproate fermentation from fruit and vegetable waste via anaerobic microbial consortia. Bioresour. Technol. 284, 398-405. doi: 10.1016/j.biortech.2019.03.124

Conflict of Interest: The authors declare that the research was conducted in the absence of any commercial or financial relationships that could be construed as a potential conflict of interest.

Copyright (C) 2020 Sabater, Ruiz, Delgado, Ruas-Madiedo and Margolles. This is an open-access article distributed under the terms of the Creative Commons Attribution License (CC BY). The use, distribution or reproduction in other forums is permitted provided the original author(s) and the copyright owner(s) are credited and that the original publication in this journal is cited, in accordance with accepted academic practice. No use, distribution or reproduction is permitted which does not comply with these terms. 